\title{
Correction to: A Tertiary-Care/Primary-Care Partnership Aimed at Improving Care for People with Eating Disorders
}

\author{
Lea Thaler ${ }^{1,2,10}$ (1) . Shiri Freiwald ${ }^{1} \cdot$ Chloe Paquin Hodge $\mathrm{e}^{1,2} \cdot$ Émilie Fletcher $^{1} \cdot$ Danaelle Cottier $^{1} \cdot$ Esther Kahan $^{1}$. \\ Erika Rossi $^{1,3} \cdot$ Myra Piat $^{2,4,5} \cdot$ Shalini Lal ${ }^{6,7,8} \cdot$ Mimi Israel $^{1,2} \cdot$ Howard Steiger $^{1,2,9}$
}

Published online: 6 July 2018

○) Springer Science+Business Media, LLC, part of Springer Nature 2018

\section{Correction to: Community Mental Health Journal https://doi.org/10.1007/s10597-018-0290-4}

The original version of this article unfortunately contained a mistake in EAT-26 values under "Patients Receiving Treatment for an ED in their Sector" section.

The correct sentence is as follows:

In addition, patients had significantly lower scores (i.e. less symptoms) at the end of treatment on the EAT-26 total score $(M$ pre $=27.56, S D=14.3 ; M$ post $=16.64$, $S D=12.22)(t(43)=4.93, \mathrm{p}=.000)$, and on depressive symptoms, as measured by the CES-D, from pre $(M=1.26, S D=0.63)$ to post-treatment $(M=0.99$, $S D=0.57)(t(43)=3.00, \mathrm{p}=.005)$.

The original article can be found online at https://doi.org/10.1007/ s10597-018-0290-4.

Lea Thaler

lea.thaler@douglas.mcgill.ca

1 Eating Disorders Continuum, Douglas Mental Health University Institute, Montreal West Island Integrated University Health and Social Service Centre (IUHSSC), Montreal, QC, Canada

2 Psychiatry Department, McGill University, Montreal, QC, Canada

3 Psychology Department, Université du Québec à Montréal, Montreal, QC, Canada

4 Douglas Institute Clinical Activities, Knowledge Transfer and Teaching Directorate, Montreal West Island Integrated University Health and Social Service Centre (IUHSSC), Montreal, QC, Canada

5 School of Social Work, McGill University, Montreal, QC, Canada
6 School of Rehabilitation, Faculty of Medicine, Université de Montréal, Montreal, QC, Canada

7 Carrefour de l'innovation et de l'évaluation en santé, Centre de recherche du Centre hospitalier de l'Université de Montréal (CRCHUM), Montreal, QC, Canada

8 PEPP-Montreal and ACCESS Open Minds, Douglas Mental Health University Institute, Montreal West Island Integrated University Health and Social Service Centre (IUHSSC), Montreal, QC, Canada

9 Psychology Department, McGill University, Montreal, QC, Canada

10 Eating Disorders Continuum, Douglas Mental Health University Institute, 6875 LaSalle Blvd, Montreal, QC H4H 1R3, Canada 